\title{
Comparison of two internationally recognized methods for determining the sound absorption coefficient
}

\author{
Pavel Drabek ${ }^{1, *}$ \\ ${ }^{1}$ Tomas Bata University in Zlin, Faculty of Applied Informatics, Department of Automation and Control Engineering, Nad Stranemi \\ 4511, Czech Republic
}

\begin{abstract}
With today's growing focus on noise control issues and the emergence of sound quality as an important aspect of product design, acoustic material testing is becoming increasingly relevant to engineers, designers and manufacturers from a broad range of industries. Acoustic material testing is the process by which acoustic characteristics of materials are determined in terms of absorption, reflection, impedance, admittance and transmission loss. Many different methods can be used to determine the acoustic properties of materials. There are standing wave method, two-microphone free field and multimicrophone techniques, reverberation chamber method, in-situ methods and many other alternative methods based on measurement of internal properties of porous materials. The point is that some methods are preferred over others because the environment in which they are measured is closer to real practice. The article deals with the comparison of the results of the measurement in the reverberation chamber and in the interferometer.
\end{abstract}

\section{Introduction}

In recent years, great emphasis has been put on improving the quality of the environment and the wellbeing of a person [1]. Hence, the proposals for new production technologies and materials must take into account all factors that may have a negative impact on the environment. For this reason, it is necessary to implement a set of measures to eliminate the harmful effects. These measures are realized on the basis of experimental measurements of characteristic physical quantities and their evaluation. One of these environmental factors is noise and the protection against its excessive exposure is an object of engineering interest.

In the case of performing an acoustic assessment of the space in order to improve the sound transmission, it is necessary to work with the values of the sound absorption coefficient $\alpha$ of the absorbent materials in relation to the frequency.

Determination of the absorption coefficient of materials for sound absorption and absorbent structures is commonly performed according to two basic methods [1][2]. The first one is a standing waves method, which allows the determination of the coefficient only for the sound waves impinging perpendicularly. The advantage of using this method is that the material impedance can be detected simultaneously [1]. However, the values found in this way have little relevance in technical practice because when the material is placed in enclosures its sound absorption coefficient is considerably different due to the omnidirectional sound waves incidents [3]. Hence its values can not be used to design acoustic adjustments, and preference is given to the results from the reverberation chamber measurement because its internal environment is closer to the final practical application. From the same assumption, other alternative methods have been developed that have a different accuracy of results, time and financial demands on the necessary equipment, for example [4][5].

The problem is that the laboratories that carry out the measurements are following a certain piece of legislation (ASTM or ISO). The point is that these documents differ in several requirements for measuring space, used equipment and the sample itself. For example, a comparison of these two approaches is mentioned in [6]. The intention of this paper is to provide information on the different measurement techniques and its differences in results.

\section{Description of the tested materials}

This point specifies the properties of two materials that were selected to compare the results of the $\alpha$ measurement coefficient in the interferometer and the reverberation chamber. The intent was to choose the elements which are characterized by different texture, porosity, thickness and the technological process of its production.

The first sample was the mineral wool bonded with organic resin ROCKWOOL - Multirock Rockmin (see Fig. 1) insulating wadding in the form of boards with dimensions $1000 \times 600 \times 40 \mathrm{~mm}$. These boards are mainly used for thermal, noise and fire insulation of roofs, ceilings and walls.

* Corresponding author: pdrabek@fai.utb.cz 


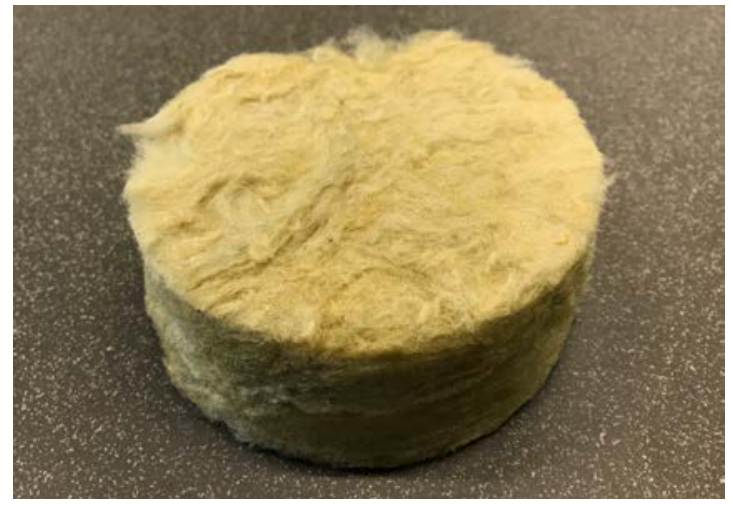

Fig. 1. Pattern of Rockwoll - Multirock Rockmin (sample 1).

The second specimen was the NOISEFLEX Classic (see Fig. 2) acoustic panel with dimensions of $1000 \times 500 \times 50 \mathrm{~mm}$. This panel is made of the Basotect material, which is a flexible foam with an open cell structure made of melamine resin. It is used in a wide range of applications, such as sound and heat insulation of buildings and vehicles, as well as cleaning sponges.

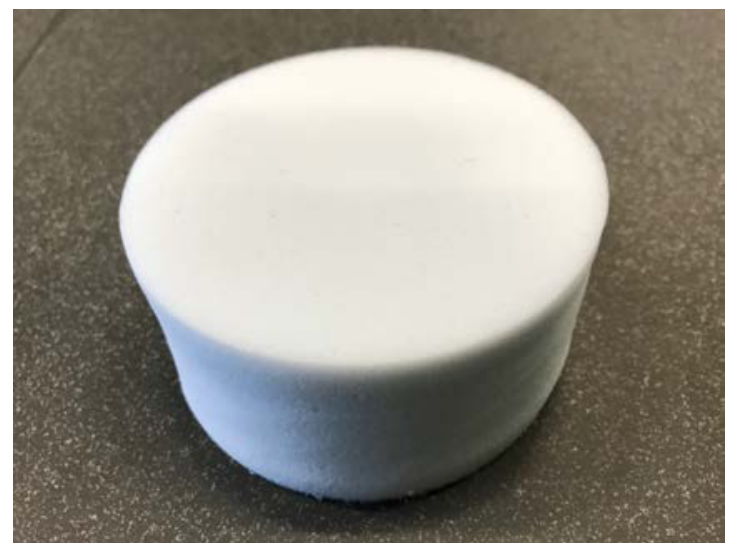

Fig. 2. Pattern of Noiseflex Classic (sample 2).

\section{Methodology}

Measurement of the above-mentioned samples was done by both methods, specific activities are described in followings.

\subsection{Impedance or standing wave tube measurement}

The principle of measuring the sound absorption factor of materials using this method is well known and is described in detail in [1]. The measurements were performed using the two-microphone tube Brüel \& Kjaer 4206 and the three-channel PULSE multi-analyzer Brüel \& Kjaer type 3560-B-030. These kits meet the standards ISO 10534-2, ASTM E1050 - 12. The measuring system consists of two cylindrical form tubes of diameter 30 and $100 \mathrm{~mm}$, which allow measuring in the frequency range $0 \mathrm{~Hz}$ to $1.6 \mathrm{kHz}$ and $500 \mathrm{~Hz}$ to $6.4 \mathrm{kHz}$, and a broadband loudspeaker that generates noise. With a broadband signal propagating from the loudspeaker, a frequency response function is measured between the two phasematched condenser microphones which are placed at set distances along the length of the tube. Each measured response must be phase calibrated according to the procedure presented in the governing standard. Afterwards, the resulting absorption curve is then determined by the combined calculation over the entire frequency range. Equations (1) and (2) define the algorithm for the computation of the sound absorption coefficient, $\alpha$, from $H$.

$$
R(j \omega)=\frac{H_{12}-e^{-j k z}}{e^{j k z}-H_{12}} e^{j 2 k(l+z)}
$$

where $H_{12}=p\left(z_{2}\right) / p\left(z_{1}\right)$ is the transfer function which is simply the ratio of pressures $p, k=2 \pi f / c$ is the wave number, $s$ is the center-to-center distance between the microphones, $z_{1}$ is the distance from the face of the test sample to the center of the nearest of the microphone, $z_{2}$ is the distance for the farther microphone, and $R(j w)$ is the complex reflection coefficient. The resulting $\alpha$ value is then given by the following equation.

$$
\alpha=1-|R(j \omega)|^{2} .
$$

Both materials were cut to the inner dimension of each tube. However, the carving itself needs to be done very accurately. It was hypothesized that any small difference between the diameter of the material sample and that of the inside of the tube would cause measurement errors [1]. This effect should be more visible in the narrower tube since small differences in diameter result in large differences in boundary pressure loading. In part, the question of the effect of these inaccuracies on the resulting values of the sound absorption coefficient is solved here [7].

\subsection{Reverberation chamber method}

As in the previous case, the reverberation chamber method is well known and does not need to be described here [2]. It is described in detail in standards [8] and [9]. The measurements were performed in a reverberation chamber at Tomas Bata University in Zlín, and the values were further compared with those obtained from a certified laboratory in Prague. At this point, it is worth to mention that the reverberation chamber in Zlín does not meet the parameters of the standards for sound absorption measurements and the measurements made in it are only indicative and serve the purpose of its further verification, which has already been carried out and published here [10]. The reverberation chamber in Zlin has a rectangular shape and volume of $93.66 \mathrm{~m}^{3}$. To eliminate the standing waves generation and to create a partial diffusion environment, the walls and the ceiling are fitted with diffusion elements. The reverberation chamber in Prague was built according to the requirements of the standards concerned. It has a volume of $200 \mathrm{~m}^{3}$ and also contains diffusion elements.

The test element was asymmetrically mounted directly on the floor of the chamber so that no part of it was closer than $1 \mathrm{~m}$ from any boundary wall (see Fig. 3). The sample was rectangular with a width/length ratio of 
between 0.7 and 1 . The area of the sample was about 9 $\mathrm{m}^{2}$. The outer flanks of the test specimen were enclosed by an acoustically reflective frame. Subsequently, the reverberation time with the sample and the empty room was measured.

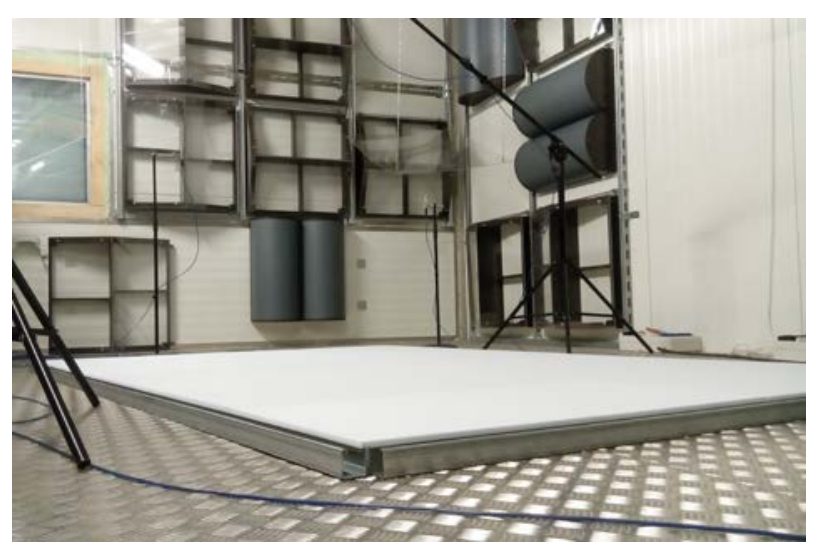

Fig. 3. Measurement of sound absorption of sample 2 in reverberation chamber at TBU in Zlin.

In the same way, both samples were measured in a reverberation chamber in Prague. The area of the samples was about $12 \mathrm{~m}^{2}$. The reverberation times were measured over a range of $100 \mathrm{~Hz}$ to $5 \mathrm{kHz}$ and was determined on the basis of the impulse response, so the chamber was excitation by a pistol shot. The source was normally placed in the corner of a room, pointing into the corner, because it maximally excites the modes of the room and reduces the amount of direct radiation from the source to the test sample. Despite these measures, a diffuse field is not completely achieved, and consequently, the reverberation time is position dependent [1]. For this reason, it is normal to use multiple sources and receiver positions and to average the results to reduce the effect of nondiffuseness [2]. Even with all these measures, the measured absorption coefficients are often more inaccurate at low than high frequencies due to modal effects.

During the measurement, microclimatic conditions were also recorded to correctly determine the amount of absorbed sound in the interior of the chamber. The procedure for calculating the attenuation factor is given in the standard [11].

\section{Results}

This point summarizes the results of the two different comparisons. The first one shows the differences between the results of the sound absorption coefficient obtained by different methods, and the second one compares the results from measurements performed in two different environments, where the first space meets the standard requirements for the volume and shape of the room and the other does not meet these requirements. These anticoincidences will be discussed in the following.

As can be seen from the Fig. 4 and 5, the $\alpha$ values determined in the reverberation chamber are higher in the entire frequency range. It is mainly because the impedance tube measures the absorption only for the perpendicular incidence of sound waves and furthermore, the absorption coefficients determined in reverberation chambers are commonly exaggerated by diffraction effects and care must be taken, where it is critical, to correctly take such effects into account. The appropriate proof can be that absorption coefficient tables published in the literature often contain values greater than 1 [1]. These results only confirm this fact.

Sample 2 displays better absorption capabilities, especially at lower frequencies, where, in particular, the higher material thickness is applied. Towards higher frequencies, the increase in absorption in both samples is very similar. In the case of impedance tube results, it can be observed that absorption increases in sample 2 much earlier. This can be caused by several factors such as, for example, a better cellular structure of the material and the method of joining the individual layers. However, it is also possible that the sample was not completely cut off and the resulting leakage affected the results.

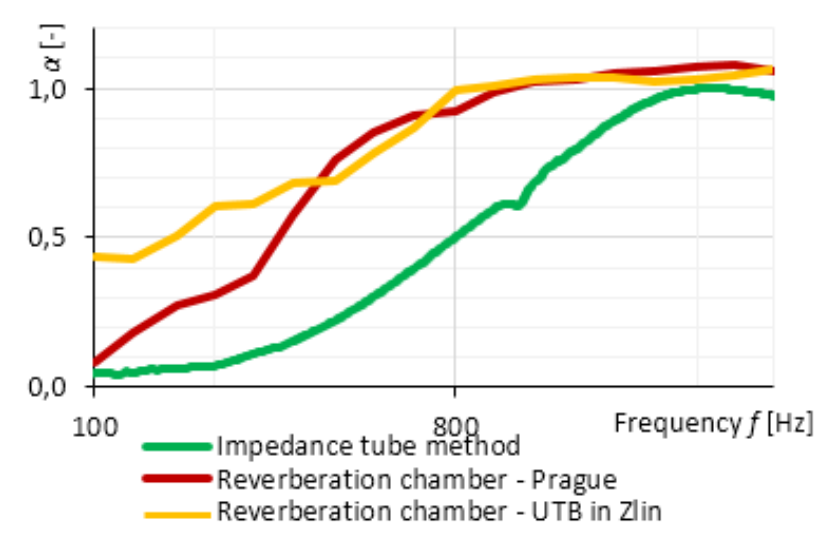

Fig. 4. Comparison of absorption curves of sample 1 for different measurement methods.

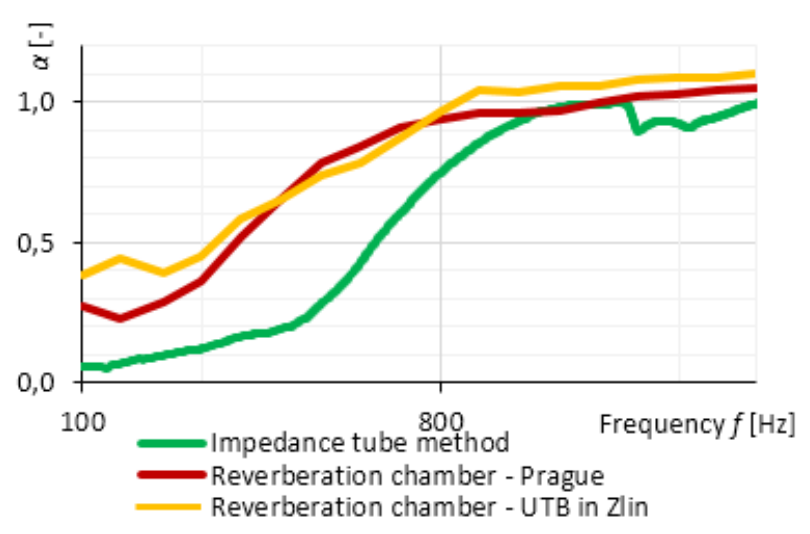

Fig. 5. Comparison of absorption curves of sample 2 for different measurement methods.

When comparing the results from both chambers, some deviations are noticeable, especially at lower frequencies. In the case of the TBU Chamber, significant fluctuations can be observed. This is due to the fact that the volume is not sufficient and the sound field is not sufficiently distributed at lower frequencies. This causes that the dispersion of reverberation times is relatively high and the uncertainty of the measurement increases 
considerably. And this is precisely why the internal chamber volume is required to be at least $150 \mathrm{~m} 3$. At higher frequencies, the values are met and the difference here is mainly due to the diffraction effect.

\section{Conclusion}

In most applications, the sound will be incident on an absorptive material from a multitude of incident angles at once. The random incidence absorption coefficient is the parameter used most in the design of spaces to specify the absorption performance of materials. The reverberation chamber test requires large sample sizes and a specialist test room and so it is expensive to undertake. For this reason, developers of absorptive materials will often use the impedance tube to build up an understanding of the material properties of small samples, before undertaking reverberation tests.

From the above, it is clear that it is very necessary to know what method can be used to obtain the desired results. For the development of new materials, the impedance tube method is suitable; however, for design acoustic adjustments of the space, the alpha coefficient obtained from the rectangular chamber measurements is much more relevant.

\section{Acknowledgement}

This work was supported by the Ministry of Education, Youth and Sports of the Czech Republic within the National
Sustainability Programme project No. LO1303 (MSMT7778/2014) and also by the Internal Grant Agency of Tomas Bata University in Zlin under the project No. IGA/CebiaTech/2017/002.

\section{References}

1. T. Cox, P. D'Antonio, Acoustic Absorbers and Diffusers, 2, (2009)

2. M. Möser, G. Müller, Handbook of Engineering Acoustics, (2013)

3. R. Novy, Hluk a chveni, (2009)

4. K. Kleinschmidt, Building Acoustics, 6, 63-70 (1999)

5. O. Robin, A. Berry, O. Doutres, N. Atalla, Acoustical Society of America, 136, 13-19 (2014)

6. M. Bischel, K. Roy, J. Greenslade, Acoustics08 Paris, AA03, 1669-1674 (2008)

7. A. Barnard, M. Rao, NOISE-CON, N/A, 1-8 (2004)

8. ASTM C423-90a, Standard, ASTM International, West Conshohocken, 2002

9. EN ISO 354, Standard, European Committee for Standardization, Brusel, (2003)

10. P. Drabek, M. Zalesak, WSEAS Transactions on Environment and Development, 12, 377-385 (2016)

11. EN ISO 9613-1, Standard, European Committee for Standardization, Brusel, (1995) 